\title{
Preparation of Dicalcium Phosphate Anhydrous (Monetite) Biological Coating on Titanium by Spray-Drying Method
}

\author{
Xingling Shi, ${ }^{1,2}$ Chao Zhao, ${ }^{3}$ Lingli Xu, ${ }^{1}$ and Qingliang Wang ${ }^{4}$ \\ ${ }^{1}$ School of Materials Science and Engineering, Jiangsu University of Science and Technology, Zhenjiang 212003, China \\ ${ }^{2}$ TusPark (Kunshan) Development Co., Ltd., Kunshan 215347, China \\ ${ }^{3}$ Hitachi Automotive System (Guangzhou) Ltd., Guangzhou 511300, China \\ ${ }^{4}$ School of Materials Science and Engineering, China University of Mining and Technology, Xuzhou 221116, China
}

Correspondence should be addressed to Lingli Xu; xulingli311@hotmail.com

Received 30 June 2017; Accepted 12 October 2017; Published 2 November 2017

Academic Editor: Charles C. Sorrell

Copyright (c) 2017 Xingling Shi et al. This is an open access article distributed under the Creative Commons Attribution License, which permits unrestricted use, distribution, and reproduction in any medium, provided the original work is properly cited.

\begin{abstract}
Titanium and its alloys have been widely used in the manufacture of endosseous implants due to excellent biocompatibility, low elastic modulus, and good chemical stability. However, the titanium based metals are essentially bioinert materials. In order to improve their bioactivity, biological coatings are usually applied. Recent studies found that, compared with hydroxyapatite coating, dicalcium phosphate anhydrous (DCPA, Monetite) coating maybe more bioactive due to higher solubility and release of Ca and P ions. In this work, DCPA coating was prepared through a novel and simple method. Briefly, high concentration DCPA solution was sprayed onto superhydrophilic titanium and the specimens were dried fast in an air-circulating oven. After repeating the process over 100 times, a compact coating was fabricated. The microstructure, chemical composition, wettability, and in vitro bioactivity of the coating were analyzed and evaluated. Results showed that the coating fully covered the substrate and consisted of a large number of uniformly sized DCPA granules that packed together closely. The coating showed good wettability and could keep the property for a long time. After immersion in simulated body fluid for 2 weeks, a large amount of bone-like apatite with low crystallinity was induced implying a good bioactivity.
\end{abstract}

\section{Introduction}

Titanium (Ti) and its alloys are important metal materials for the manufacture of load bearing bone implants due to excellent corrosion resistance, biocompatibility, and lower elasticity modulus. However, the Ti based metals are essentially bioinert materials and are insufficient in osseointegration ability. Instead of biological fixation, their combination with human bones mainly depends on mechanical locking. Moreover, a fibrous capsule intervening at the interface of implant and bone is always formed after the implantation, especially when a relatively smooth implant was used $[1,2]$. Such kind of fibrous capsule is fragile and its adhesion to both implant and surrounding bone cavity is quite low, and therefore it is easy to peel or form crush induced necrosis under stress and micromovement. In this case, a serious secondary infection is likely to happen and may lead to a premature loss of reconstruction function of the implants. In addition, the fibrous capsule is poorly differentiated and may undergo hyperplasia or transform into tumor tissue under sustained mechanical stimulation. Thus, a fast and strong bonding to the host bone without soft tissue intervention is a prerequisite for the long-term and stable performance of $\mathrm{Ti}$ implants.

Bioactive coatings play an important role in improving the osseointegration of metal implant with bone and, among them, the hydroxyapatite (HA) coating is the most widely used [3-5]. However, HA is not osteoinductive and when the implantation site is poor in bone mass and density, such as osteoporosis, even HA coated implants cannot form a rapid biological integration with the host bone. With the increasing desire from patients for early loading and rapid healing, it is urgent to prepare new biological implant coatings with osteoinductivity. By contrast, acidic calcium phosphates, dicalcium phosphate anhydrous $\left(\mathrm{CaHPO}_{4}, \mathrm{DCPA}\right.$, Monetite) and dicalcium phosphate dehydrate $\left(\mathrm{CaHPO}_{4} \cdot \mathrm{H}_{2} \mathrm{O}, \mathrm{DCPD}\right.$, 
Brushite), are more degradable at physiologic $\mathrm{pH}$ than $\mathrm{HA}$ and will form a $\mathrm{Ca}^{2+}$ and $\mathrm{PO}_{4}{ }^{3-}$ supersaturated zone at the interface of implant and bone that may induce osteoblastic differentiation [6]. Among them, DCPA has gained great attention recently as it showed degradation and regeneration properties superior to DCPD. For instance, in vitro evaluation showed that DCPA ensured active osteoclast resorption and would also induce higher levels of osteogenic genes expression of bone marrow cells than DCPD $[7,8]$. In vivo studies are even more promising. Torres and coworkers grafted DCPA and autologous bone granules separately into critical sized bone defects in rabbit calvaria and after 8 weeks they found that the volumes of mineralized tissue formed by two kinds of granules were not significantly different $[9,10]$. Oryan et al. compared the effectiveness of calcium phosphates/chitosan-gelatin-platelet gel scaffold on the healing of critical size radial bone defects in rats 8 weeks after implantation. They reported that DCPA filler accelerated bone regeneration and provided a much better balance between substitute resorption and new bone formation compared with $\beta$-TCP and HA [11]. DCPA degrades at a mild rate and, unlike $\beta$-TCP and DCPD, it will not undergo phase transition into HA in physiologic environment [12]. Such unique properties may bring about its superiority in terms of resorption and remodeling. Therefore, we think DCPA coatings are expected to be a new generation of bioactive coatings used on surface of metal endosseous implants.

Several techniques have been developed for preparation of DCPA coating. Kuroda et al. heated the Ti substrate up to $150^{\circ} \mathrm{C}$ in a solution with calcium and phosphorus ions by large alternating current (up to $25 \mathrm{~A}$ ). Water gasified vigorously on Ti surface and DCPA was formed. However, the coating grew in the form of lamellar and brick-like clusters and failed to completely cover the substrate [13]. Ortiz et al. prepared DCPD on metal substrate by electrochemical deposition and chemical deposition separately and then heated the specimens to get DCPA coating. In this procedure, $\mathrm{Ca} / \mathrm{P}$ ratio was difficult to control as spontaneous grain growth happened during the formation of DCPD coating and therefore the final products might contain calcium pyrophosphate (CPPD) which to some extent weakens the biocompatibility of coatings $[14,15]$. DCPA coating can also be prepared by sol-gel method. However, such method not only consumes time as always, but also induces calcium carbonate and other impurities [16]. Obviously, DCPA coatings by these methods can not meet the requirement of biomedical applications. Moreover, the current methods present several drawbacks such as complicated equipment, high safety standard, high current or temperature operation, complicated process, low efficiency, and high energy consumption. Besides, the DCPA granules by these methods usually scatter on substrate surface as clusters and could not cover the substrate completely. Nevertheless, the impurity phase generated during preparation may impair the properties of coating.

In this work, a novel DCPA coating preparation method was proposed based on the dissolution characteristic of dicalcium phosphate. Briefly, high concentration DCPA aqueous solution was prepared and then a DCPA coating was prepared

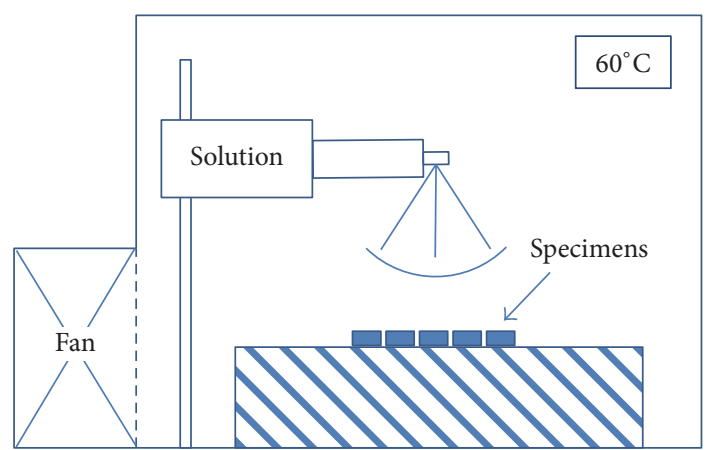

FIGURE 1: Illustration of coating preparation progress.

on superhydrophilic Ti substrate by repeated spray-drying treatment. The microstructure, chemical composition, wettability, and in vitro apatite inducing ability of the coating were analyzed and evaluated.

\section{Materials and Methods}

2.1. Pretreatment of Specimens. Annealed commercially pure Ti pieces (Grade 2, Baoji Titanium Industry Company Ltd., China), cut into $10 \mathrm{~mm} \times 10 \mathrm{~mm} \times 2.5 \mathrm{~mm}$, were used as substrates. The specimens were wet-polished with 280\#, 400\#, and 800\# SiC abrasive paper successively and then ultrasonically washed in acetone and distilled water for $10 \mathrm{~min}$, respectively. After being dried in air, these specimens were pretreated by acid etching to get superhydrophilic surface. Briefly, the $\mathrm{Ti}$ specimens were submerged in a mixture of $98 \%$ (wt) $\mathrm{H}_{2} \mathrm{SO}_{4}, 37 \%$ (wt) $\mathrm{HCl}$, and distilled water with the volume ratio of $5: 2: 3$ and etched for $30 \mathrm{~min}$. After that, the specimens were sufficiently washed with tap water, neutralized by diluted $\mathrm{NaHCO}_{3}$ solution, and then ultrasonically washed for $10 \mathrm{~min}$ in distilled water. The pretreated specimens were placed in distilled water before use.

2.2. Solution Preparation and Coating Fabrication. High concentration DCPA aqueous solutions, $11.5 \mathrm{~g} / \mathrm{L}$ (unsaturated), were prepared by dissolving analytically pure DCPA powder (Sinopharm Chemical Reagent Co. Ltd., China) into diluted $\mathrm{HCl}$ solution of $0.15 \mathrm{~mol} / \mathrm{L}$ with the assistance of ultrasonicator. Pretreated Ti specimens were taken out of distilled water, placed into an air-circulating oven set at $60^{\circ} \mathrm{C}$. Immediately after drying, the specimens were repeatedly sprayed with DCPA solution for certain times by a programmable automatic electric sprayer (TS-T, Shanghai Kezhe Biochemical Technology Co., Ltd., China). The spraying distance was $3 \mathrm{~cm}$, the spray radius was $4 \mathrm{~cm}$, and the spray volume was $0.1 \mathrm{ml}$ per time. A interval of 2 min was set between two successive sprays to ensure complete drying of the specimen surface. The experiment setup is illustrated in Figure 1. After the final spray and drying, specimen were washed thoroughly with distilled water and then were finally dried in the oven for $30 \mathrm{~min}$.

2.3. Characterizations. Product phases and crystallinity were investigated by X-ray diffraction (XRD, D/MAX-3B, Rigaku 


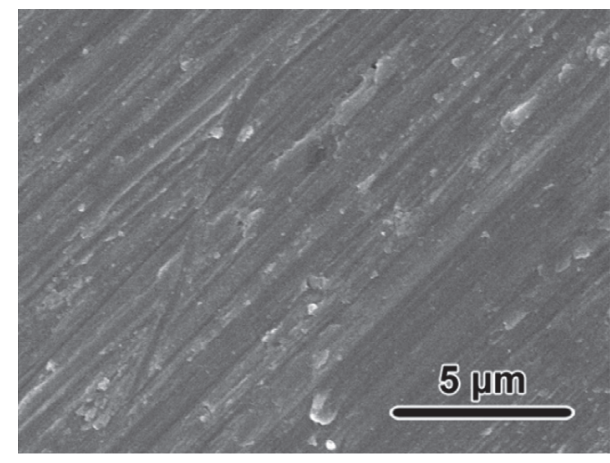

(a)

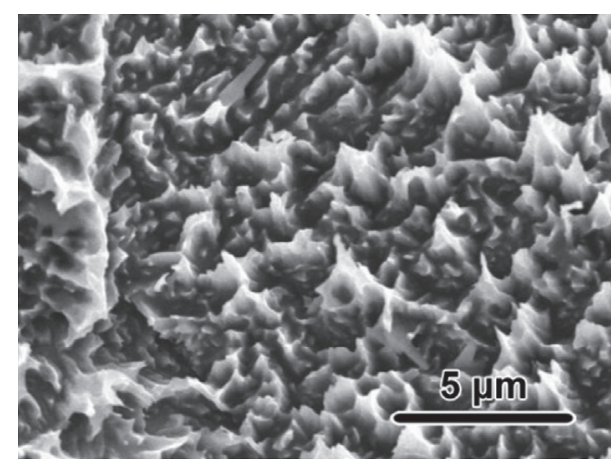

(b)

FiguRE 2: Surface morphology of specimens before and after pretreatment: (a) sandpaper polished; (b) acid etched specimens.
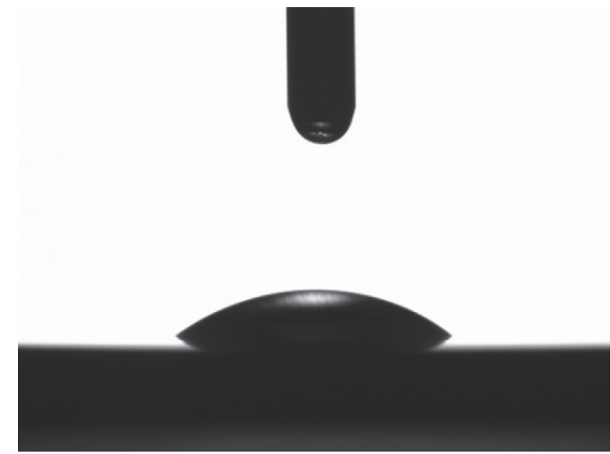

(a)
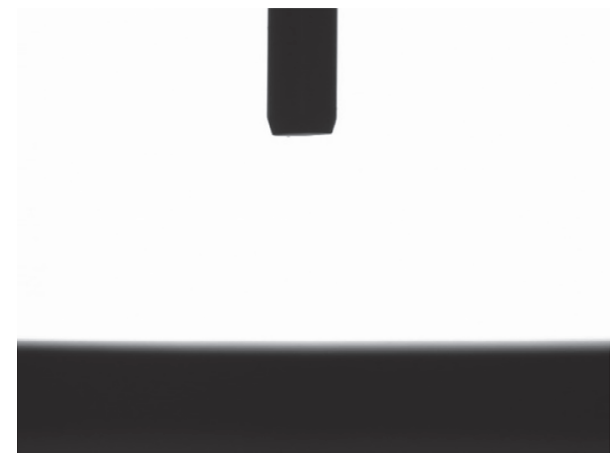

(b)

FIGURE 3: Contact angles of specimens before and after pretreatment: (a) sandpaper polished; (b) acid etched specimens.

Co., USA). The surface morphologies of the specimens were observed with scanning electronic microscope (SEM, S-3000, Hitachi Co., Japan). Surface roughness of specimens was analyzed by laser scanning confocal microscopy (VKX100, Keyence Co., Japan). The contact angle of distilled water on DCPA coating was measured by the static contact angle measuring instrument (JC2000A, Shanghai Zhongchen Digital Technic Apparatus Co., Ltd., China). Bioactivity of DCPA coating was evaluated through the simulated body fluid (SBF) immersion test. The main ion concentrations of the SBF were nearly equal to that of human plasma and the $\mathrm{pH}$ was adjusted to 7.4 by trihydroxymethyl aminomethane (Tris) and $1.0 \mathrm{~mol} / \mathrm{L} \mathrm{HCl}$ solution [17]. DCPA coated specimens were immersed in $1.0 \mathrm{~mol} / \mathrm{L} \mathrm{NaOH}$ solution at $60^{\circ} \mathrm{C}$ for $1 \mathrm{~h}$ and after thorough rinse they were immersed in SBF for 14 days in an incubator set at $37^{\circ} \mathrm{C}$. After that, specimens were taken out, rinsed with distilled water, and dried in air at room temperature. And then the immersed specimens were analyzed by SEM and XRD.

\section{Results and Discussions}

3.1. Pretreatment. Surface morphologies of specimens before and after pretreatment were shown in Figure 2. After acid etching, texture of scratches generated during the wet abrasion had disappeared and submicron sized etch pits were found distributing all over the surface. The surface roughness was found increased, from $0.26 \mu \mathrm{m}$ to $1.61 \mu \mathrm{m}$, after acid etching treatment. Surface wettability of specimens was obviously improved as shown in Figure 3. The contact angle on polished surface was about $37^{\circ}$, whereas the water drop spread on acid etched surface fast and the contact angle became $0^{\circ}$. It meant that acid etching had imposed superhydrophilicity to the $\mathrm{Ti}$ specimens. However, such excellent wettability gradually degraded when specimens were kept in ambient air. As shown in Figure 4, the contact angle (CA) increased with the increase of storage time. After 14 days storage it was over $100^{\circ}$. In order to preserve the superhydrophilicity, specimens were submersed in distilled water in a sealed glass bottle. As it could be seen, the CA was still $0^{\circ}$ after 14 days immersion in distilled water.

\subsection{Characterizations of DCPA Coating}

3.2.1. Surface Morphology. Figure 5 showed the SEM observation results of acid etched Ti specimens after spray-drying treatment with different repeating times. At the beginning, when the number of repetitions was low, round shaped clumps were formed on the surface, as shown in Figure 5(a). They were separated, occupying one or more etch pits, but closely attached to the specimens surface. However, the acid etched surface was still visible, because of the limited 


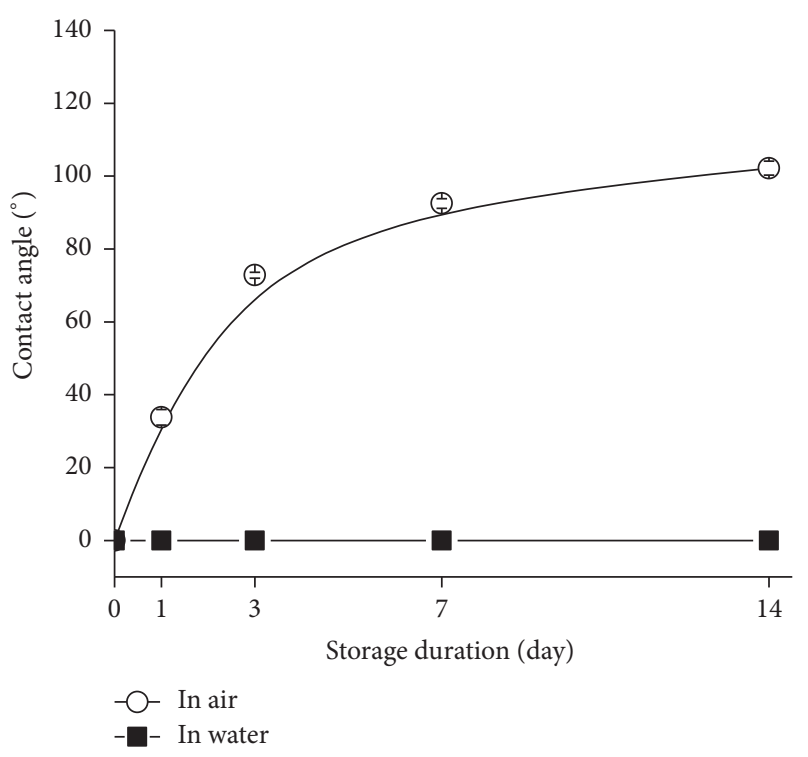

FIGURE 4: The changes of contact angles with increasing storage duration.

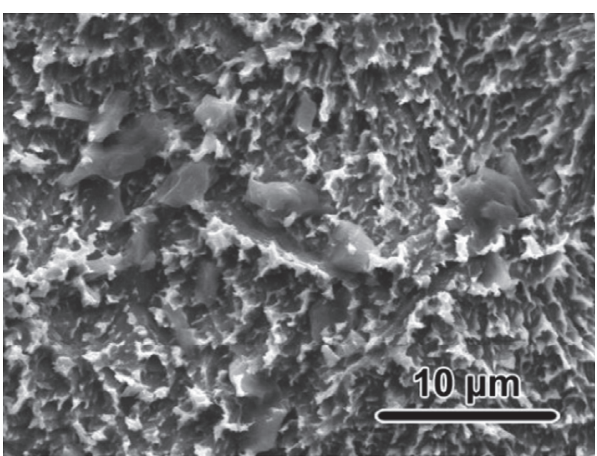

(a)

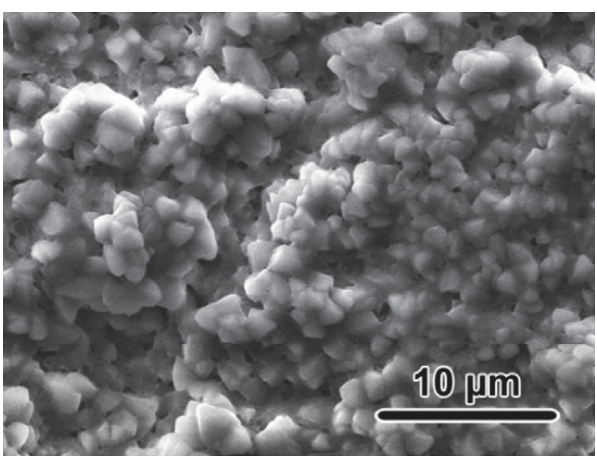

(c)

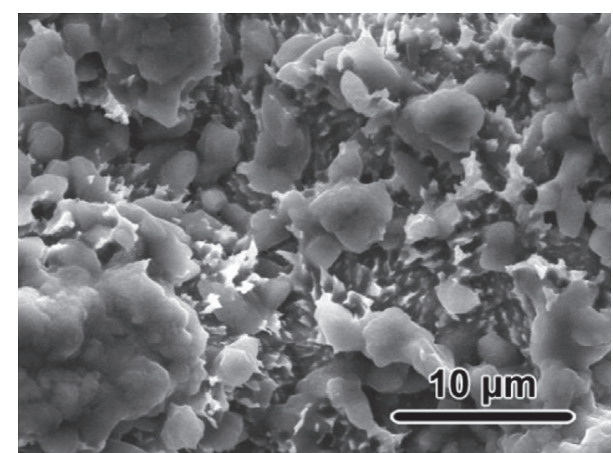

(b)

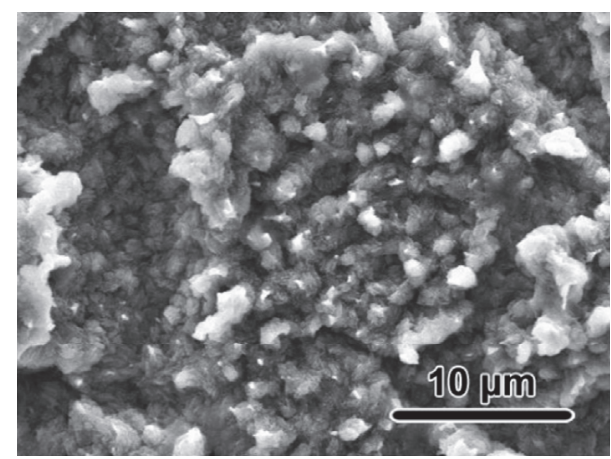

(d)

FIGURE 5: Surface morphology changes with increasing spray-drying repetition number: (a) 20 times; (b) 60 times; (c) 100 times; (d) 140 times.

numbers of these particles. As the spray-drying operation repeated, more round clumps were formed, and they started to pile up and contact each other, as shown in Figure 5(b). When the repeating times increase to 100 , the whole specimens surface was covered with numerous cubic granules of about $1.5 \mu \mathrm{m}$ in diameter and the acid etched pits disappeared.
These granules stacked up tightly with each other and had formed a compact layer, Figure 5(c). The later XRD result confirmed that these are DCPA particles. However, further increase in repeating times, up to 140 , induced a less compact coating with particles showing a hairy surface, as shown in Figure 5(d). 


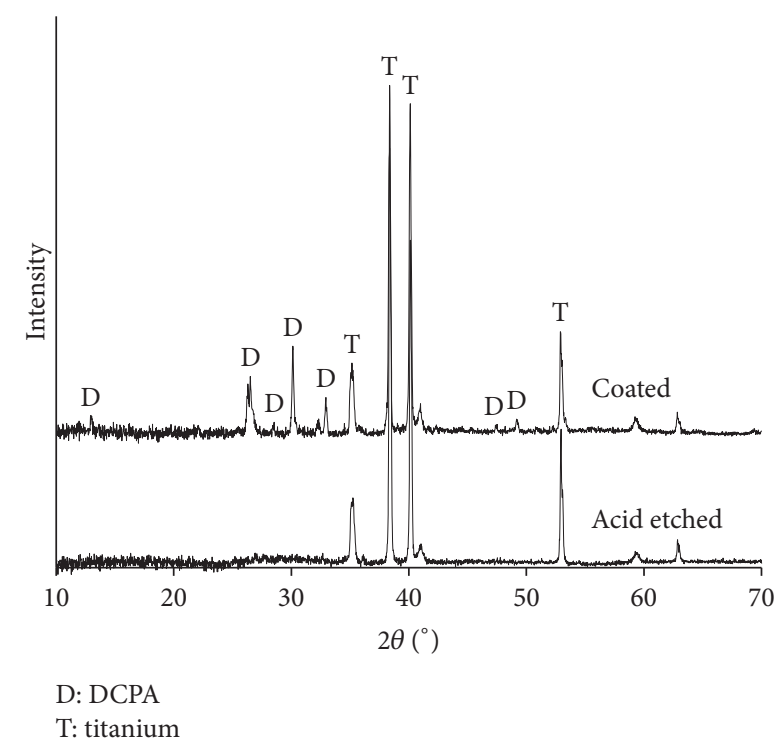

FIGURE 6: XRD patterns of specimens after 100 times of spray-drying treatment.

3.2.2. Coating Phase Analysis. Figure 6 showed the XRD patterns of acid etched Ti after 100 times of spray-drying treatment. It could be seen that several diffraction peaks appeared besides those from pure Ti substrate and all these new peaks could be ascribed to DCPA (PDF \#01-077-0128). Among them, the three most intense ones, located at $2 \theta=$ $26.4^{\circ}, 30.2^{\circ}$, and $32.8^{\circ}$, could be assigned to the (220), (112), and (102) planes, respectively. The diffraction results have excluded existence of by-products in the coating as peaks from other crystalized Ca-P compound and amorphous phases were not found. Besides, all the diffraction peaks were sharp in shape which indicated well crystallized structures and an atomic ratio that was close to the ideal stoichiometry. According to calculations using Scherrer formula, the crystal in the cubic granules was $40.59 \mathrm{~nm}$ in average. Thermodynamically, the product of Ca-P precipitation from solution could be predicted based on the diagram of Ca-P compounds solubility versus $\mathrm{pH}$. When the $\mathrm{pH}$ was less than 4.0, DCPA crystals could preferentially be precipitated from the liquid if the ions were supersaturated $[6,18]$. Thus, in this method, low solution $\mathrm{pH}$ was the controlling factor for the pure DCPA product.

It could be inferred that, after each spraying, the former precipitated granules on substrate would be dissolved and precipitated again during the following drying stage. With the increase of repeating times and the accumulation of solute, a compact DCPA coating would be formed gradually. Compared with that by Kuroda et al., the DCPA coating prepared by this spray-drying method could cover the acid etched surface completely and the granules in the coating were uniform in size, contacted with each other more closely according to SEM observation $[13,19]$. The superhydrophilic surface facilitated even and fast evaporation of water once a thin liquid layer was formed by spraying and was thought to be an important precondition for the homogenous precipitation of DCPA granules. However, as water evaporated quickly, once a compact coating had been formed, the following sprays would only induce partial dissolution of these granules and thus the top surface of the coating became hairy as the spray-drying repeating times reached 140 .

3.2.3. Wettability. The wettability of DCPA coating was evaluated by measuring the contact angle, as shown in Figure 7. Same as acid etched specimens, the freshly prepared DCPA coated ones also showed superhydrophilicity. Moreover, after being stored in ambient air for 4 weeks, it still kept an excellent hydrophilicity with the contact angle only rising about $15^{\circ}$. These results showed that, the DCPA coating not only inherited superhydrophilicity of acid etched surface but also preserved a good wettability after a long storing duration.

Currently, surface of Ti implants designed for osseointegration are usually roughened by sandblast and/or acid etching. Submicron scaled pits generated on the Ti surface mimic the microstructure of natural bone and facilitate osteogenic differentiation of stem cells. Besides, such roughening could impose superhydrophilicity to surface of implant which greatly improves the bioactivity of Ti. For instance, the protein in the body fluid could be rapidly adsorbed to the implant surface at the implantation moment and show conformation favorable for cell attachment, proliferation, and osteogenic differentiation [20]. However, a fresh acid etched surface also possesses high surface energy, and therefore carbon containing molecules in the ambient air or packages will easily be adsorbed onto its surface and keep accumulating as storage time increases. Such contamination impairs the excellent wettability and degrades osteoconductivity of rough implants and has become a universal problem that challenges both the producer and surgeons [21-24]. This study demonstrated that hydrophilicity of the bone implants with rough surface could be preserved by slightly soluble bioactive coatings and hence the bioactivity degradation will be overcome.

3.2.4. In Vivo Apatite Induction. The surface morphology observation and XRD results of DCPA coated specimens (spray-drying 100 times) after immersion in SBF for 2 weeks were shown in Figures 8 and 9. As could be seen from the SEM image, the whole surface of specimens was covered by spherical crystal clusters after immersion. It was the typical surface morphology of bioactive materials after SBF immersion test $[25,26]$. In the XRD pattern peaks ascribed to apatite could be observed. Meanwhile, it should be noticed that apatite was of low crystallinity and signals of amorphous phase were found at the diffraction angles from $20^{\circ}$ to $40^{\circ}$. In addition, the diffraction peaks of DCPA remained clearly and their relative intensities versus those of Ti substrate did not seem to decrease. It indicated that the DCPA coating did not undergo obvious degradation during the immersion. The SBF immersion experiment tallies with in vivo animal experiment very well and is reproducible and easy to be operated, so it is widely used to evaluate the bioactivity of materials. The spontaneous deposition of apatite from SBF within 2 weeks indicated that the DCPA coating fabricated by this method 

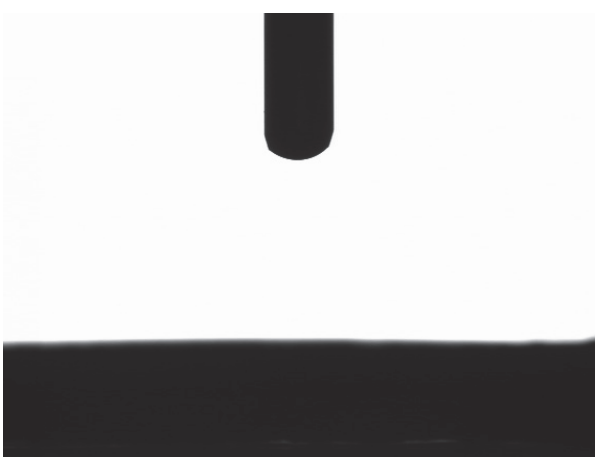

(a)
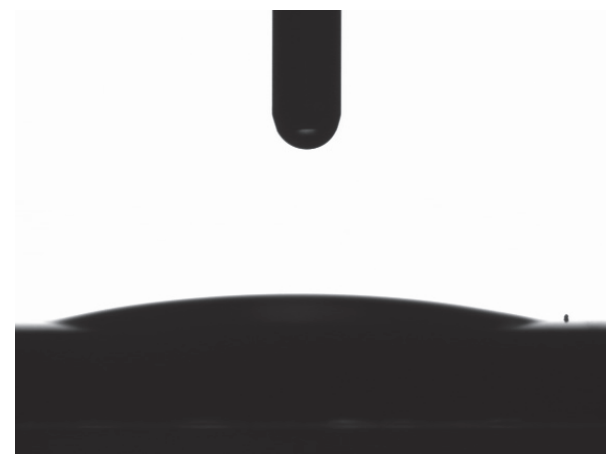

(b)

FIGURE 7: Contact angles of DCPA coatings: (a) freshly prepared; (b) after 4 weeks storage in air.

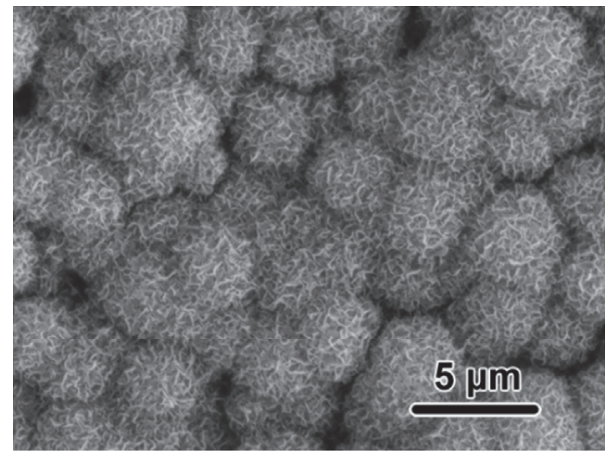

FIGURE 8: Surface morphology of coated specimens after being immersed in SBF for 2 weeks.

has good bioactivity and it is hopeful of inducing new bone to grow rapidly on the surface of implants and increasing the initial stability of implants.

\section{Conclusion}

DCPA coating was fabricated on titanium substrate through a novel and simple spray-drying method. The coating was compact, fully covered substrate and was consist of uniform sized DCPA granules that packed together closely. The coating showed good wettability and could keep the property for a long time. After immersion in simulated body fluid for 2 weeks, a large amount of bone-like apatite with low crystallinity was induced on the coating surface, which implied good bioactivity. This method is feasible and efficient, and it is expected to be used for other substrates as long as the surface showed good hydrophilicity.

\section{Conflicts of Interest}

The authors declare that they have no conflicts of interest.

\section{Acknowledgments}

The authors gratefully acknowledge the financial support from National Natural Science Foundation of China (51275514),

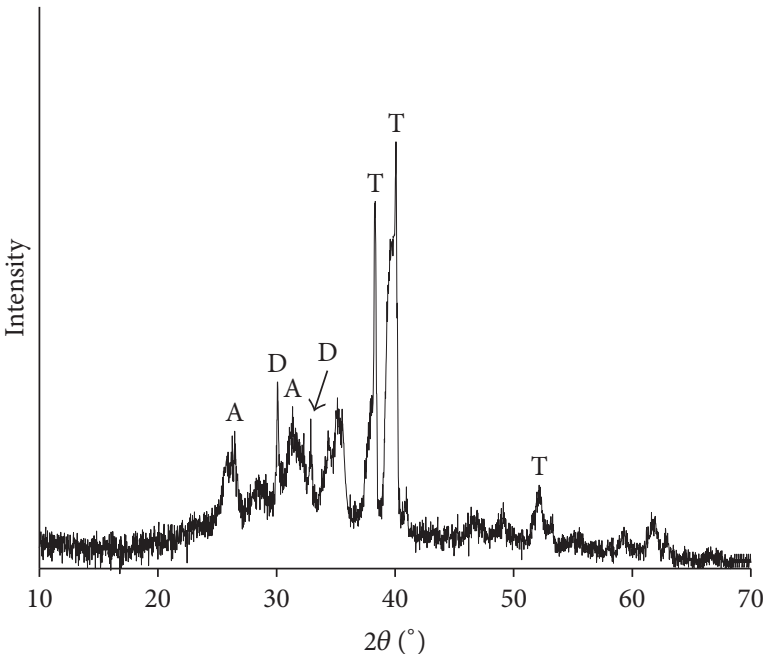

A: apatite
D: DCPA
T: titanium

FIGURE 9: Phase analysis of coated specimens after being immersed in SBF for 2 weeks.

Natural Science Foundation of Jiangsu Province (BK20160566), China Postdoctoral Science Foundation (2016M601754), and Jiangsu Planned Projects for Postdoctoral Research Funds (1421601085B).

\section{References}

[1] L. Le Guéhennec, A. Soueidan, P. Layrolle, and Y. Amouriq, "Surface treatments of titanium dental implants for rapid osseointegration," Dental Materials, vol. 23, no. 7, pp. 844-854, 2007.

[2] B. Elliott and T. Goswami, "Implant material properties and their role in micromotion and failure in total hip arthroplasty," International Journal of Mechanics and Materials in Design, vol. 8, no. 1, pp. 1-7, 2012.

[3] X. Shi, L. Xu, T. B. Le et al., "Partial oxidation of TiN coating by hydrothermal treatment and ozone treatment to improve its osteoconductivity," Materials Science and Engineering C: Materials for Biological Applications, vol. 59, pp. 542-548, 2016. 
[4] S. R. Paital and N. B. Dahotre, "Calcium phosphate coatings for bio-implant applications: materials, performance factors, and methodologies," Materials Science and Engineering: R: Reports, vol. 66, no. 1-3, pp. 1-70, 2009.

[5] A. Haider, S. Haider, S. S. Han, and I.-K. Kang, "Recent advances in the synthesis, functionalization and biomedical applications of hydroxyapatite: a review," RSC Advances, vol. 7, no. 13, pp. 7442-7458, 2017.

[6] K. Ishikawa, S. Matsuya, Y. Miyamoto, and K. Kawate, "9.05bioceramics," in Comprehensive Structural Integrity, 2003.

[7] M. Montazerolghaem, M. Karlsson Ott, H. Engqvist, H. Melhus, and A. J. Rasmusson, "Resorption of monetite calcium phosphate cement by mouse bone marrow derived osteoclasts," Materials Science and Engineering C: Materials for Biological Applications, vol. 52, pp. 212-218, 2015.

[8] F. Tamimi, D. L. Nihouannen, H. Eimar, Z. Sheikh, S. Komarova, and J. Barralet, "The effect of autoclaving on the physical and biological properties of dicalcium phosphate dihydrate bioceramics: Brushite vs. monetite," Acta Biomaterialia, vol. 8, no. 8, pp. 3161-3169, 2012.

[9] T. Jesús, T. Iskandar, C. A. Jatsue et al., "Monetite granules versus particulate autologous bone in bone regeneration," Annals of Anatomy, vol. 200, pp. 126-133, 2015.

[10] F. Tamimi, J. Torres, U. Gbureck et al., "Craniofacial vertical bone augmentation: a comparison between 3D printed monolithic monetite blocks and autologous onlay grafts in the rabbit," Biomaterials, vol. 30, no. 31, pp. 6318-6326, 2009.

[11] A. Oryan, S. Alidadi, and A. Bigham-Sadegh, "Dicalcium phosphate anhydrous: an appropriate bioceramic in regeneration of critical-sized radial bone defects in rats," Calcified Tissue International, vol. 101, no. 5, pp. 530-544, 2017.

[12] Z. Sheikh, Y. L. Zhang, L. Grover, G. E. Merle, F. Tamimi, and J. Barralet, "In vitro degradation and in vivo resorption of dicalcium phosphate cement based grafts," Acta Biomaterialia, vol. 26, pp. 338-346, 2015.

[13] K. Kuroda, Y. Miyashita, R. Ichino, M. Okido, and O. Takai, "Preparation of calcium phosphate coatings on titanium using the thermal substrate method and their in vitro evaluation," Materials Transactions, vol. 43, no. 12, pp. 3015-3019, 2002.

[14] I. Y. Ortiz, A. Raybolt dos Santos, A. M. Costa et al., "In vitro assessment of zinc apatite coatings on titanium surfaces," Ceramics International, vol. 42, no. 14, pp. 15502-15510, 2016.

[15] K. Li, B. Wang, B. Yan, and W. Lu, "Microstructure in vitro corrosion and cytotoxicity of Ca-P coatings on ZK60 magnesium alloy prepared by simple chemical conversion and heat treatment," Journal of Biomaterials Applications, vol. 28, no. 3, pp. 375-384, 2013.

[16] H. Eshtiagh-Hosseini, M. R. Houssaindokht, M. Chahkandhi, and A. Youssefi, "Preparation of anhydrous dicalcium phosphate, DCPA, through sol-gel process, identification and phase transformation evaluation," Journal of Non-Crystalline Solids, vol. 354, no. 32, pp. 3854-3857, 2008.

[17] L. Xu, X. Shi, C. Ouyang, and W. Liu, "In vitro apatite formation, protein adsorption and initial osteoblast responses on titanium surface enriched with magnesium," Rare Metal Materials and Engineering, vol. 46, no. 6, pp. 1512-1517, 2017.

[18] H. Oonishi and H. Oomamiuda, "Chapter 9 degradation/ resorption in bioactive ceramics in orthopaedics," in Handbook of Biomaterial Properties, Springer, New York, NY, USA, 1998.

[19] A. V. Zavgorodniy, R. S. Mason, R. Z. LeGeros, and R. Rohanizadeh, "Adhesion of a chemically deposited monetite coating to a Ti substrate," Surface and Coatings Technology, vol. 206, no. 21, pp. 4433-4438, 2012.

[20] X. Shi, L. Xu, M. L. Munar, and K. Ishikawa, "Hydrothermal treatment for TiN as abrasion resistant dental implant coating and its fibroblast response," Materials Science and Engineering C: Materials for Biological Applications, vol. 49, pp. 1-6, 2015.

[21] W. Att, N. Hori, F. Iwasa, M. Yamada, T. Ueno, and T. Ogawa, "The effect of UV-photofunctionalization on the time-related bioactivity of titanium and chromium-cobalt alloys," Biomaterials, vol. 30, no. 26, pp. 4268-4276, 2009.

[22] H. Minamikawa, W. Att, T. Ikeda, M. Hirota, and T. Ogawa, "Long-term progressive degradation of the biological capability of titanium," Materials, vol. 9, no. 2, p. 102, 2016.

[23] X. Shi, L. Xu, K. B. Violin, and S. Lu, "Improved osseointegration of long-term stored SLA implant by hydrothermal sterilization," Journal of the Mechanical Behavior of Biomedical Materials, vol. 53, pp. 312-319, 2016.

[24] X. Shi, L. Xu, Q. Wang, Sunarso, and L. Xu, "Hydrothermal sterilization improves initial osteoblast responses on sandpaperpolished titanium," Materials, vol. 10, no. 7, article 812, 2017.

[25] T. Kokubo and H. Takadama, "How useful is SBF in predicting in vivo bone bioactivity?" Biomaterials, vol. 27, no. 15, pp. 29072915, 2006.

[26] L. Xu, X. Shi, Z. Zhu, Z. Wang, and Y. Jin, "Improved in vitro osteoconductivity of pure titanium modified with magnesium by hydrothermal treatment," Rare Metal Materials and Engineering, vol. 49, no. 9, pp. 2613-2617, 2017. 

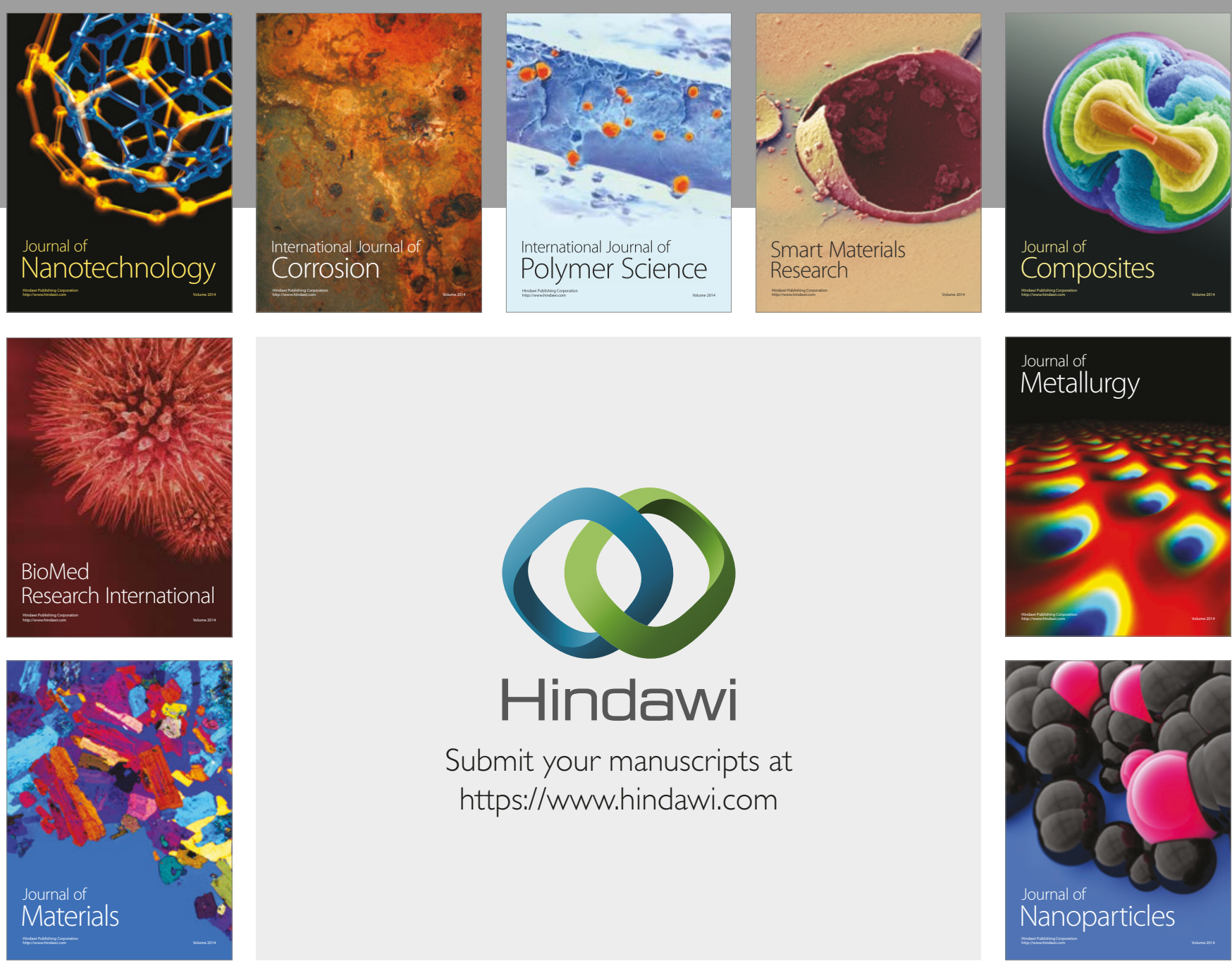

\section{Hindawi}

Submit your manuscripts at

https://www.hindawi.com
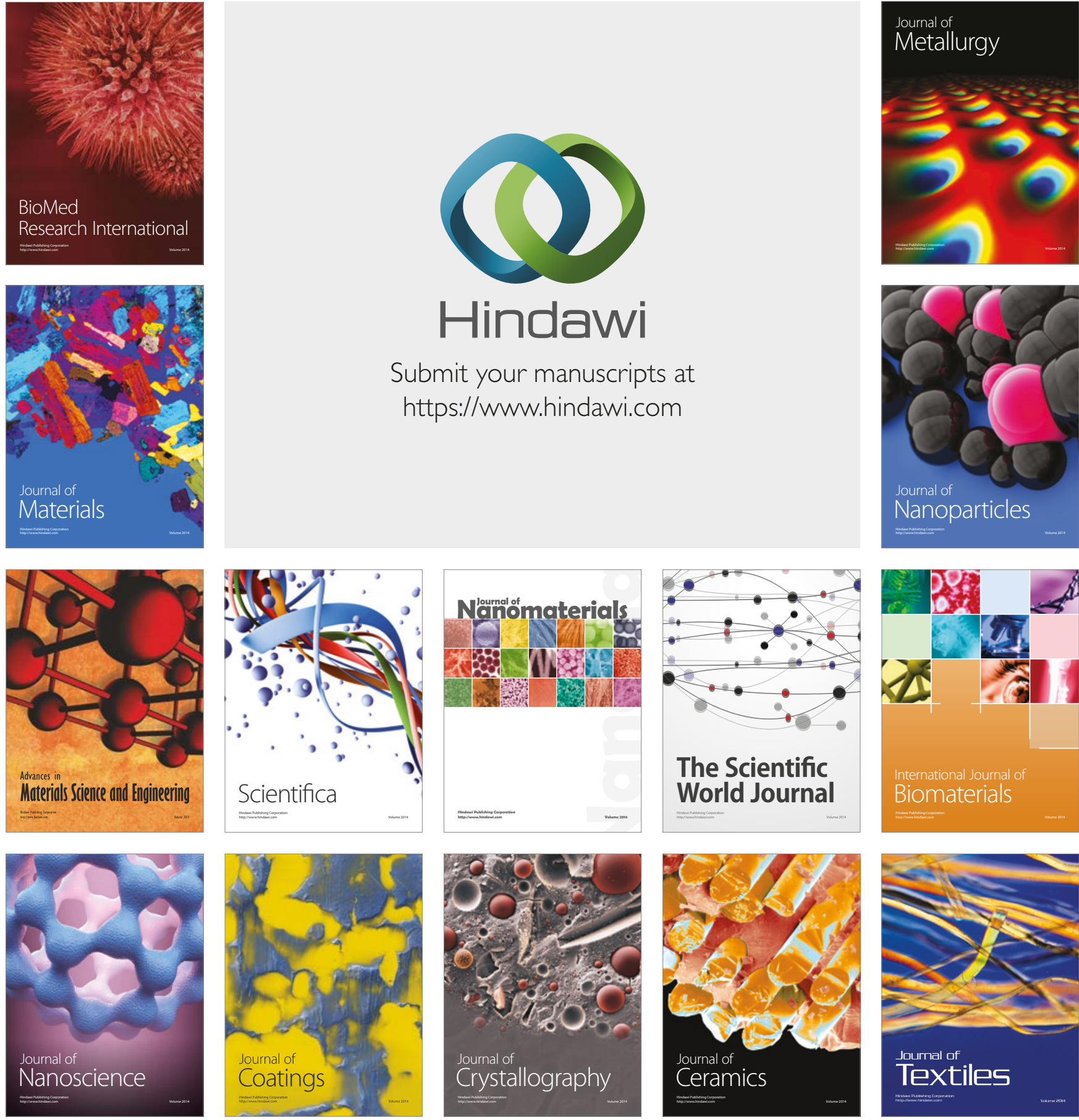

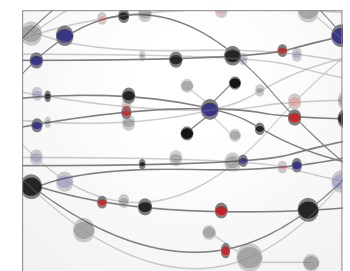

The Scientific World Journal
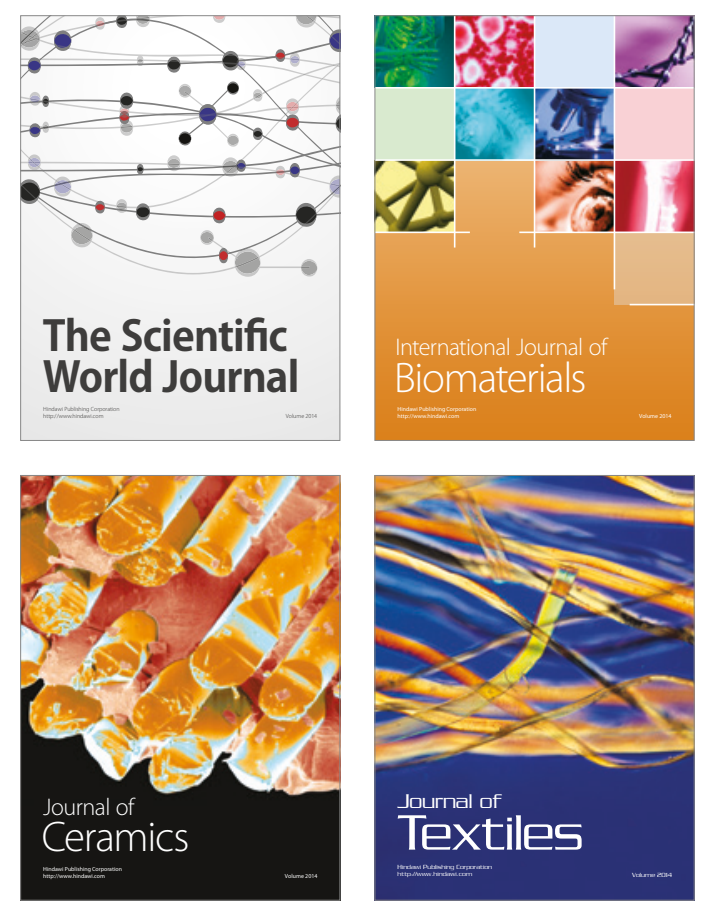